\title{
Application of FIB to Metal Alloy TEM Sample Preparation
}

\author{
Y.W Riddle, S. Shankar, M.M. Makhlouf
}

Worcester Polytechnic Institute, Advanced Casting Research Center, Worcester, MA 01609 USA

Focused ion beam milling (FIB) provides a unique solution for metallurgists who are interested in investigating very specific regions of their alloys in the transmission electron microscope (TEM). Before FIB metallurgists investigating highly heterogeneous alloys had to make many TEM samples hoping the area of interest coincided with the electron transparent region. This ad-hoc method is time consuming and one can never be absolutely sure where the specimen is extracted from in relation to the surrounding material. FIB solves this problem. Our research with a variety of Al-Si casting alloys and steels from the de Center (WTC) has benefited from FIB because we can select microstructures from exact areas of interest to study and characterize in the TEM. Furthermore we can image and analyze the general microstructure beforehand in the FIB to show exactly where the TEM sample came from, hus connecting several orders of spatial magnitude together for a more complete analysis. This is important because the scale of features in these alloys is generally much larger than a typical TEM sample. In this paper we present a case study of results from the Al-Si and steel alloy TEM samples created using the FIB.

TEM samples were milled from the bulk using a dual-beam FIB [1] to include grain boundaries and their neighboring material allowing the examination of phase composition by energy dispersive spectroscopy (EDS), microstructural morphology, and relative orientation between the various crystalline phases by electron diffraction. In general, the target thickness for each specimen was approximately $100 \mathrm{~nm}$, which is sufficiently thin for electron transparency. Subsequent to FIB milling, the extracted TEM samples were placed on standard carbon coated support grids. TEM work was performed on a JEOL $100 \mathrm{C}$ operated at $100 \mathrm{keV}$ or a Hitachi HF2000 operated at $200 \mathrm{keV}$ [2]. So far the FIB process itself has not introduced artifacts into the samples preventing analysis using conventional TEM. There is surface contamination of the samples from $\mathrm{Ga}$ ions emanating from the FIB milling. Given that Ga is neither an intentional addition nor a known impurity in any of the metal alloys of interest to our investigations, we can confidently parse its presence from microstructural and mechanistic significance.

The study of the eutectic transformation in Al-Si casting alloys and its ability to be modified by various minor additions has been the focus of much research for more than eight decades now. A recent review summarizes the current understanding of this industrially important transformation [3]. Modifying the eutectic structure affords great control over the as-solidified microstructure and thus the alloy's mechanical properties. Many speculations and theories have emerged attempting to explain the operative mechanisms of this modification but all have suffered from a lack of directly observed evidence. By creating TEM samples which span the interdendritic eutectic region into the primary grain we study the orientation relationships between eutectic silicon, eutectic aluminum, and primary aluminum to understand how nucleation of each eutectic phase occurs in both unmodified Al-7wt\%Si alloy and Al-7wt\%Si alloys modified with up to $0.022 \mathrm{wt} \% \mathrm{Sr}$. Details of sample preparation have been published elsewhere [4]. This has led to the discovery of several preferred orientation relationships in the modified alloys that do not exist in the unmodified alloy and deepens 
our understanding of the true operating mechanisms of modification. With greater understanding, we will have greater control and predictability of modification to take best advantage of this effect.

Figure 1 illustrates a typical TEM sample of Al-Si created using the FIB milling technique with comparison to the same sample imaged using the TEM. Electron diffraction patterns with known orientation from each phase are compared to diffraction patterns of neighboring grains to determine if there is a preferred orientation relationship between the two.

Analysis of WTC steel from buildings 1, 2, and 7 is ongoing. It is currently believed that the steels suffered from oxidation, sulfidation, and partial melting leading to severe hot corrosion attack along grain boundaries which greatly reduced the originally $1 / 2$ inch thick sections in many areas [5]. The interface of the hot corrosion regions and parent steel is currently being characterized to help find evidence of what temperature this material was exposed to, how the reaction occured, potential sources of the sulfur, and to aid metallurgists, engineers, and architects against the failure of such structural steels in the future. The tremendous porosity and large compositional gradients present prevent the creation of TEM samples using conventional methods. FIB milling gives us the ability to select areas of interest between the hot corrosion and parent steel regions with the only known possibility for creating useful TEM samples.

a)

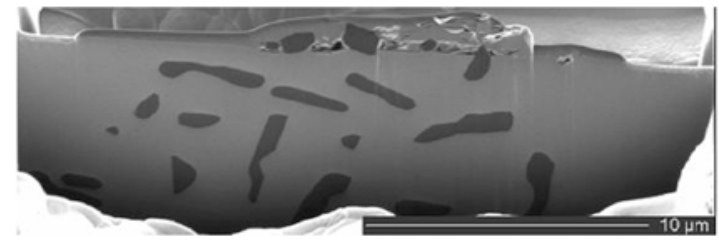

b)

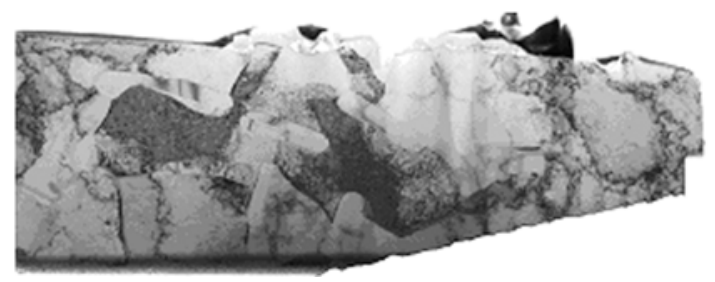

Figure 1: (a) Sample of Al-7Si wt\% created and imaged in the FIB, (b) same sample imaged in the TEM

References:

[1] FIB located at Lehigh University, Lehigh, Pennsylvania, USA.

[2] Hitachi HF2000 TEM located at Georgia Institute of Technology, Atlanta, GA, USA.

[3] M.M. Makhlouf, H.V. Guthy, J. Light Metals 1 (2001) 199.

[4] S. Shankar, Y.W. Riddle, M.M. Makhlouf, accepted for publication in Met. Trans.

[5] Private conversations with R.R. Biederman, Worcester Polytechnic Institute, Worcester, MA, USA. 